\title{
Effect of moderate running training and mindfulness- based stress reduction on immune system and quality of life in women with breast cancer receiving aromatase inhibitors
}

Johannes F Mattes*

Department of Oncology, VAMED Rehaklinik Ahrenshoop GmbH, Germany

\section{Introduction and summary}

Women with breast cancer receiving aromatase inhibitors often cannot resume normal life because their quality of life is affected severely from side effects.

At the moment these physical side effects of aromatase inhibitors cannot be avoided effectively so that a stress reduction by undergoing a mindfulness-based stress (MBSR) reduction training seems to be a chance to enhance the quality of life for affected women.

In this context our randomized control trial examines the hypothesis, whether an MBSR training of 8 weeks improves quality of life for women of the MBSR group (MBSR group $n=10$ ). By contrast women of the control group $(n=15)$ take part in Nordic walking once per week.

Surprisingly, results reveal that women of the MBSR group noticed an increase of breast pain located in their breast affected by cancer and also an increase of insomnia. The cortisol level in the afternoon had fallen slightly at the end of 8 weeks in participants of the control group and had risen in participants of the MBSR group.

However, as a long-term effect the cognitive functioning in the MBSR group was improved after 16 weeks.

\section{Methods}

Participants of the MBSR group (intervention group, limited to $\mathrm{N}=10$ ) and control group with Nordic walking training were randomized. All women had breast cancer, were aged 50-79, had hormon-rezeptive tumours, and a tumour stage UICC I-III [1].

The period of training was 8 weeks for both groups. Three measurements were taken in each group:

- $\mathrm{t} 1$ : before the beginning of 8 weeks training

- t2: 8 weeks after the beginning of training (at the end of training)

- $\mathrm{t} 3$ : 16 weeks after the beginning of training (8 weeks after training)

Primary endpoint (measuring time points $\mathrm{t} 1$ and $\mathrm{t} 2$ ) were the physiological parameters of serum cortisol and serum prolactin. Secondary endpoint was the quality of life at the measuring time points $\mathrm{t} 2$ and $\mathrm{t} 3$ according to the inventary of EORTC-questionnaire (Figure 1)

- EORTC QLQ-C30 Version 3.0 and

- EORTC QLQ-BR23

\section{Statistical methods}

Considering the small sample, we focused the effect size to get valuable information to clinical effects despite not having significance.

We did an analysis of covariance (ANCOVA) with timepoint t1 (= beginning of the intervention) as covariate.

The effect size $\eta^{2}$ was calculated related to the effect of the factor "group". For interpretation of the effect size we used table 1 [1].

\section{Results}

\section{Results of the physiological parameters}

Analysis of covariance: Timepoints $\mathrm{t} 1$ and $\mathrm{t} 2$ : Participants of the MBSR group had a small increase of postprandial cortisol at 2 p.m., whereas participants of the Nordic walking group had a small decrease of postprandial cortisol at 2 p.m. $\left(\eta^{2}=0,162\right.$, Table 2).

\section{Results of the EORTC-Questionnaires}

Analysis of covariance timepoints t1 (beginning of intervention) and $\mathrm{t} 2=8$ weeks (end of intervention/training). Participants of the MBSR group reported an increase of breast pain, table $3(\mathrm{p}=0,042$; $\left.\eta^{2}=0,175\right)$ and an increase of isomnia $\left(\eta^{2}=0,113\right.$, Table 3$)$, which disappeared 8 weeks after the end of MBSR training.

Analysis of covariance timepoints t1 (beginning of intervention) und $\mathrm{t} 3=16$ weeks ( $=8$ weeks after finishing intervention). Participants of the MBSR group had an increase of cognitive functioning $\left(\mathrm{\eta}^{2}=0,131\right.$, Table 4).

\section{Discussion}

The quality of life was not improved for participants of both groups as a short-time effect after 8 weeks of training, thus Nordic walking or MBSR training were both equally ineffective to improve quality of life.

The striking result for MBSR training was the increased sensitization for unpleasant body sensations in breasts. Accordingly,

${ }^{\star}$ Correspondence to HJohannes F Mattes, Department of Oncology, VAMED Rehaklinik Ahrenshoop GmbH, Dorfstrasse 55, 18347 Ostseebad Ahrenshoop, Germany, Tel: +49 38220 63-335; Fax: +49 38220 63-440; E-mail: johannes. mattes@vamed-gesundheit.de

Received: July 01, 2019; Accepted: July 09, 2019; Published: July 12, 2019 
Mattes JF (2019) Effect of moderate running training and mindfulness-based stress reduction on immune system and quality of life in women with breast cancer receiving aromatase inhibitors

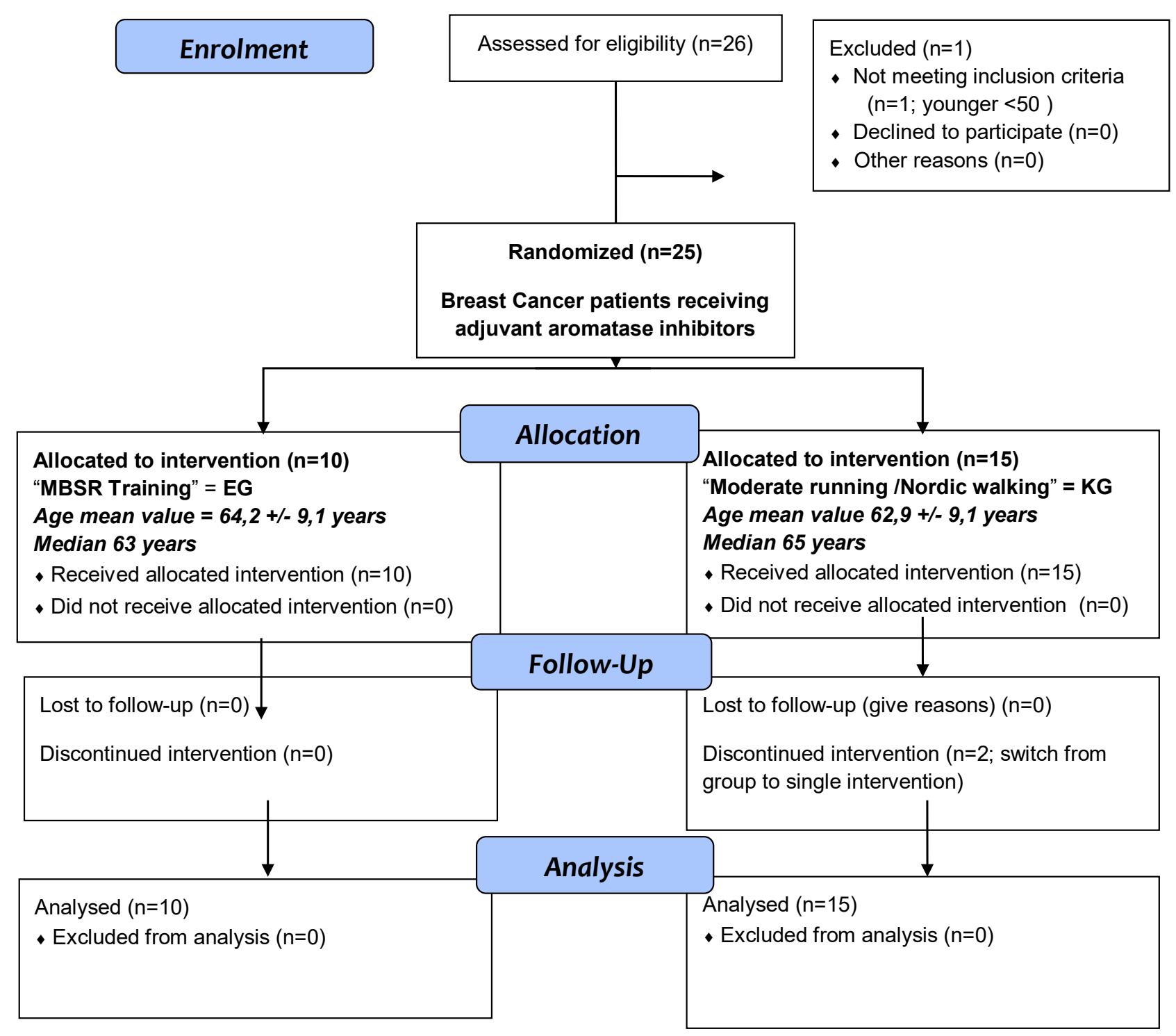

Figure 1. Consort flow chart

Table 1. Interpretation of effect size

\begin{tabular}{|c|c|c|c|}
\hline & Small & Moderate \\
\hline $\boldsymbol{r}$ & $<0.3$ & $0.3-0.5$ & Large \\
\hline $\boldsymbol{d}$ & $<0.5$ & $0.5-0.8$ & $>0.5$ \\
\hline $\mathbf{\eta}^{2}$ & 0.06 & $0.06-0.14$ & $<0-8$ \\
\hline
\end{tabular}

Table 2. Physiological parameters

\begin{tabular}{|c|c|c|c|c|c|c|}
\hline $\begin{array}{c}\text { Parameters } \\
\text { Mean values (and SD) }\end{array}$ & $\begin{array}{c}\text { t1 } \\
\text { MBSR }\end{array}$ & $\begin{array}{c}\text { t2 } \\
\text { MBSR }\end{array}$ & $\begin{array}{c}\text { t1 } \\
\text { Nordic } \\
\text { walking }\end{array}$ & $\begin{array}{c}\text { t2 } \\
\text { Nordic } \\
\text { walking }\end{array}$ & $\begin{array}{c}\text { ANCOVA } \\
(t 1 \text { = covariate })\end{array}$ & effect size* \\
\hline $\begin{array}{l}\text { Cortisol } \\
\text { at } 2 \text { pm } \\
(\mathrm{SD})\end{array}$ & $\begin{array}{c}304,2 \\
(109,309)\end{array}$ & $\begin{array}{c}318 \\
(102,177)\end{array}$ & $\begin{array}{c}279,867 \\
(151,394)\end{array}$ & $\begin{array}{c}228,533 \\
(100,904)\end{array}$ & $\begin{array}{c}\mathrm{F}=4,252 \mathrm{df}=1 \\
\mathrm{p}=0,051 \text { (group) }\end{array}$ & $\eta^{2}=0,162$ \\
\hline $\begin{array}{l}\text { Prolaktin } \\
\text { at } 2 \text { pm } \\
\text { (SD) }\end{array}$ & $\begin{array}{c}8,659 \\
(1,773)\end{array}$ & $\begin{array}{c}9,386 \\
(2,1)\end{array}$ & $\begin{array}{c}8,549 \\
(2,643)\end{array}$ & $\begin{array}{c}9,996 \\
(4,078)\end{array}$ & $\begin{array}{c}F=0,262 \mathrm{df}=1 \\
\mathrm{p}=0,614(\text { group})\end{array}$ & $\eta^{2}=0,012$ \\
\hline
\end{tabular}

*effect size related to the effect of the factor "group"

[cortisol standard value in serum: $138-690 \mathrm{nmol} / \mathrm{l}$; prolactin standard value in serum: $4,79-23.3 \mu \mathrm{g} / \mathrm{l}$ ] 
Mattes JF (2019) Effect of moderate running training and mindfulness-based stress reduction on immune system and quality of life in women with breast cancer receiving aromatase inhibitors

Table 3. Analysis of covariance: timepoints $\mathrm{t} 1$ and $\mathrm{t} 2=8$ weeks.

\begin{tabular}{|c|c|c|c|c|c|c|c|}
\hline $\begin{array}{c}\text { Parameters } \\
\text { Mean values (and } \\
\text { SD) }\end{array}$ & $\begin{array}{c}\text { t1 } \\
\text { MBSR }\end{array}$ & $\begin{array}{c}\text { t2 } \\
\text { MBSR }\end{array}$ & $\begin{array}{c}\text { t1 } \\
\text { Nordic walking }\end{array}$ & $\begin{array}{c}\mathbf{t} 2 \\
\text { Nordic walking }\end{array}$ & \multicolumn{2}{|c|}{$\begin{array}{c}\text { ANCOVA } \\
(\mathrm{t} 1 \text { = covariate })\end{array}$} & effect size* \\
\hline Quality of Life (SD) & $\begin{array}{c}53,33 \\
(16,29)\end{array}$ & $\begin{array}{c}61,67 \\
(18,92)\end{array}$ & $\begin{array}{c}65,00 \\
(21,41)\end{array}$ & $\begin{array}{c}64,44 \\
(21,47)\end{array}$ & $\mathrm{F}=1,100 \mathrm{df}=1$ (group) & $\mathrm{p}=0,306$ & $\eta^{2}=0,048$ \\
\hline $\begin{array}{c}\text { Physical } \\
\text { Functioning (SD) }\end{array}$ & $\begin{array}{c}71,33 \\
(12,98)\end{array}$ & $\begin{array}{c}74,00 \\
(13,86)\end{array}$ & $\begin{array}{c}80,44 \\
(21,30)\end{array}$ & $\begin{array}{c}81,33 \\
(18,89)\end{array}$ & $\mathrm{F}=0,004 \mathrm{df}=1$ (group) & $\mathrm{p}=0,951$ & $\eta^{2}=0,001$ \\
\hline $\begin{array}{c}\text { Role } \\
\text { Functioning (SD) }\end{array}$ & $\begin{array}{c}61,67 \\
(17,66)\end{array}$ & $\begin{array}{c}61,67 \\
(24,91)\end{array}$ & $\begin{array}{l}67,78 \\
(34,20)\end{array}$ & $\begin{array}{c}71,11 \\
(26.33)\end{array}$ & $\mathrm{F}=0,624 \mathrm{df}=1$ (group) & $\mathrm{p}=0,438$ & $\eta^{2}=0,028$ \\
\hline $\begin{array}{c}\text { Emotional } \\
\text { Functioning (SD) }\end{array}$ & $\begin{array}{c}55,83 \\
(26,95)\end{array}$ & $\begin{array}{c}61,67 \\
(24,91)\end{array}$ & $\begin{array}{c}58,89 \\
(31,88)\end{array}$ & $\begin{array}{c}70,56 \\
(26,14)\end{array}$ & $\mathrm{F}=1,383 \mathrm{df}=1$ (group) & $\mathrm{p}=0,252$ & $\eta^{2}=0,059$ \\
\hline $\begin{array}{l}\text { Cognitive } \\
\text { Functioning } \\
\text { (SD) }\end{array}$ & $\begin{array}{c}70,00 \\
(30,23)\end{array}$ & $\begin{array}{c}71,67 \\
(26,12)\end{array}$ & $\begin{array}{c}71,11 \\
(31,16)\end{array}$ & $\begin{array}{c}70,00 \\
(32,85)\end{array}$ & $\mathrm{F}=0,179 \mathrm{df}=1$ (group) & $\mathrm{p}=0,677$ & $\eta^{2}=0,008$ \\
\hline $\begin{array}{c}\text { Social } \\
\text { Functioning (SD) }\end{array}$ & $\begin{array}{c}60,00 \\
(43,18)\end{array}$ & $\begin{array}{c}71,67 \\
(27,27)\end{array}$ & $\begin{array}{c}64,44 \\
(36,11)\end{array}$ & $\begin{array}{c}70,00 \\
(34,62)\end{array}$ & $\mathrm{F}=0,216 \mathrm{df}=1$ (group) & $\mathrm{p}=0,646$ & $\eta^{2}=0,010$ \\
\hline Fatigue & $\begin{array}{c}48,89 \\
(29,26)\end{array}$ & $\begin{array}{c}46,67 \\
(27,12)\end{array}$ & $\begin{array}{c}38,52 \\
(28,75)\end{array}$ & $\begin{array}{c}36,30 \\
(29,83)\end{array}$ & $\mathrm{F}=0,058 \mathrm{df}=1$ (group) & $\mathrm{p}=0,811$ & $\eta^{2}=0,003$ \\
\hline $\begin{array}{c}\text { Nausea and } \\
\text { Vomiting (SD) }\end{array}$ & & & $\begin{array}{c}5,56 \\
(17,44)\end{array}$ & $\begin{array}{c}3,33 \\
(12,91)\end{array}$ & $\mathrm{F}=0,381 \mathrm{df}=1$ (group) & $\mathrm{p}=0,544$ & $\eta^{2}=0,017$ \\
\hline Pain (SD) & $\begin{array}{c}38,33 \\
(24,91)\end{array}$ & $\begin{array}{c}45,00 \\
(30,48)\end{array}$ & $\begin{array}{c}36,67 \\
(32,24)\end{array}$ & $\begin{array}{c}38,89 \\
(27,94)\end{array}$ & $\mathrm{F}=0,308 \mathrm{df}=1$ (group) & $\mathrm{p}=0,585$ & $\eta^{2}=0,014$ \\
\hline Dyspnoea (SD) & $\begin{array}{c}23,33 \\
(22,50)\end{array}$ & $\begin{array}{c}20,00 \\
(28,11)\end{array}$ & $\begin{array}{c}33,33 \\
(37,80)\end{array}$ & $\begin{array}{c}28,89 \\
(27,79)\end{array}$ & $\mathrm{F}=0,090 \mathrm{df}=1$ (group) & $p=0,768$ & $\eta^{2}=0,004$ \\
\hline Insomnia (SD) & $\begin{array}{c}56,67 \\
(38,65)\end{array}$ & $\begin{array}{c}60,00 \\
(37,84)\end{array}$ & $\begin{array}{c}57,78 \\
(36,66)\end{array}$ & $\begin{array}{c}44,44 \\
(39,17)\end{array}$ & $\begin{array}{c}F=2,792 \mathrm{df}=1 \\
\text { (group) }\end{array}$ & $\mathbf{p}=\mathbf{0 , 1 0 9}$ & $\eta^{2}=\mathbf{0 , 1 1 3}$ \\
\hline Appetite loss (SD) & $\begin{array}{c}20,00 \\
(35,83)\end{array}$ & $\begin{array}{c}16,67 \\
(36,00)\end{array}$ & $\begin{array}{c}11,11 \\
(24,12)\end{array}$ & $\begin{array}{c}6,67 \\
(18,69)\end{array}$ & $\mathrm{F}=0,262 \mathrm{df}=1$ (group) & $\mathrm{p}=0,614$ & $\eta^{2}=0,012$ \\
\hline Constipation (SD) & $\begin{array}{c}13,33 \\
(32,20)\end{array}$ & $\begin{array}{c}20,00 \\
(35,83)\end{array}$ & $\begin{array}{c}22,22 \\
(27,22)\end{array}$ & $\begin{array}{c}17,78 \\
(24,77)\end{array}$ & $\mathrm{F}=1,113 \mathrm{df}=1$ (group) & $\mathrm{p}=0,303$ & $\eta^{2}=0,048$ \\
\hline Diarrhoea (SD) & $\begin{array}{c}3,33 \\
(10,54)\end{array}$ & $\begin{array}{c}3,33 \\
(10,54)\end{array}$ & $\begin{array}{c}6,67 \\
(18,69)\end{array}$ & $\frac{0,00}{(0,00)}$ & $\mathrm{F}=2,391 \mathrm{df}=1$ (group) & $\mathrm{p}=0,136$ & $\eta^{2}=0,098$ \\
\hline $\begin{array}{l}\text { Financial } \\
\text { Diffuculties } \\
\quad(\mathrm{SD})\end{array}$ & $\begin{array}{c}30,00 \\
(42,89)\end{array}$ & $\begin{array}{c}20,00 \\
(32,20)\end{array}$ & $\begin{array}{c}26,67 \\
(38,21)\end{array}$ & $\begin{array}{l}(24,44 \\
(38,76)\end{array}$ & $\mathrm{F}=1,502 \mathrm{df}=1$ (group) & $\mathrm{p}=0,233$ & $\eta^{2}=0,064$ \\
\hline Body Image (SD) & $\begin{array}{c}52,50 \\
(35,15)\end{array}$ & $\begin{array}{c}59,17 \\
(35,67)\end{array}$ & $\begin{array}{c}62,78 \\
(36,31)\end{array}$ & $\begin{array}{c}66,11 \\
(37,86)\end{array}$ & $\mathrm{F}=0,078 \mathrm{df}=1$ (group) & $\mathrm{p}=0,782$ & $\eta^{2}=0,004$ \\
\hline $\begin{array}{c}\text { Sexual } \\
\text { Functioning (SD) }\end{array}$ & $\begin{array}{c}77,08 \\
(30,78)\end{array}$ & $\begin{array}{c}78,57 \\
(31,50)\end{array}$ & $\begin{array}{c}72,22 \\
(33,73)\end{array}$ & $\begin{array}{c}76,19 \\
(25,08)\end{array}$ & $\mathrm{F}=0,285 \mathrm{df}=1$ (group) & $\mathrm{p}=0,600$ & $\eta^{2}=0,016$ \\
\hline $\begin{array}{c}\text { Sexual } \\
\text { Enjoyment (SD) }\end{array}$ & $\begin{array}{c}66,67 \\
(47,14)\end{array}$ & $\begin{array}{c}66,67 \\
(47,14)\end{array}$ & $\begin{array}{c}50,00 \\
(43,03)\end{array}$ & $\begin{array}{c}66,67 \\
(27,22)\end{array}$ & \multicolumn{2}{|c|}{ not possible to evaluate } & \\
\hline $\begin{array}{c}\text { Future } \\
\text { Perspective (SD) }\end{array}$ & $\begin{array}{c}50,00 \\
(28,33)\end{array}$ & $\begin{array}{c}53,33 \\
(32,20)\end{array}$ & $\begin{array}{c}46,67 \\
(41,40)\end{array}$ & $\begin{array}{c}35,56 \\
(36,66)\end{array}$ & $\mathrm{F}=2,427 \mathrm{df}=1$ (group) & $\mathrm{p}=0,134$ & $\eta^{2}=0,099$ \\
\hline $\begin{array}{c}\text { Systemic } \\
\text { Therapy } \\
\text { Side Effects (SD) }\end{array}$ & $\begin{array}{c}34,76 \\
(19,83)\end{array}$ & $\begin{array}{c}29,96 \\
(22,07)\end{array}$ & $\begin{array}{c}27,94 \\
(14,94)\end{array}$ & $\begin{array}{c}26,03 \\
(17,90)\end{array}$ & $\mathrm{F}=1,043 \mathrm{df}=1$ (group) & $\mathrm{p}=0,318$ & $\eta^{2}=0,045$ \\
\hline $\begin{array}{l}\text { Breast } \\
\text { Symptoms } \\
\text { (SD) }\end{array}$ & $\begin{array}{c}15,83 \\
(19,02)\end{array}$ & $\begin{array}{c}25,00 \\
(19,25)\end{array}$ & $\begin{array}{l}25,00 \\
(21,13)\end{array}$ & $\begin{array}{c}22,22 \\
(21,52)\end{array}$ & $\mathrm{F}=4,663 \mathrm{df}=1$ (group) & $p=0,042$ & $\eta^{2}=0,175$ \\
\hline $\begin{array}{l}\text { Arm Symptoms } \\
\text { (SD) }\end{array}$ & $\begin{array}{c}33,33 \\
(29,63)\end{array}$ & $\begin{array}{c}35,56 \\
(30,45)\end{array}$ & $\begin{array}{c}37,04 \\
(27,11)\end{array}$ & $\begin{array}{c}31,48 \\
(21,99)\end{array}$ & $\mathrm{F}=1,610 \mathrm{df}=1$ (group) & $\mathrm{p}=0,218$ & $\eta^{2}=0,068$ \\
\hline $\begin{array}{l}\text { Upset by Hair } \\
\text { loss (SD) }\end{array}$ & 67,67 & $\begin{array}{c}22,22 \\
(38,49)\end{array}$ & $\begin{array}{c}61,11 \\
(38,97)\end{array}$ & $\begin{array}{c}41,67 \\
(42,72)\end{array}$ & $\mathrm{F}=1,812 \mathrm{df}=1$ (group) & $\mathrm{p}=0,236$ & $\eta^{2}=0,266$ \\
\hline
\end{tabular}

*effect size related to the effect of the factor "group" 
Mattes JF (2019) Effect of moderate running training and mindfulness-based stress reduction on immune system and quality of life in women with breast cancer receiving aromatase inhibitors

Table 4. Analysis of covariance: timepoints $t 1$ and $\mathrm{t} 3=16$ weeks.

\begin{tabular}{|c|c|c|c|c|c|c|c|}
\hline $\begin{array}{c}\text { Parameters } \\
\text { Mean values (and } \\
\text { SD) }\end{array}$ & $\begin{array}{c}\text { t1 } \\
\text { MBSR }\end{array}$ & $\begin{array}{c}\mathbf{t 3} \\
\text { MBSR }\end{array}$ & $\begin{array}{c}\text { t1 } \\
\text { Nordic } \\
\text { walking }\end{array}$ & $\begin{array}{c}\text { t3 } \\
\text { Nordic } \\
\text { walking }\end{array}$ & \multicolumn{2}{|c|}{$\begin{array}{c}\text { ANCOVA } \\
(\mathrm{t} 1=\text { covariate })\end{array}$} & effect size* \\
\hline Quality of Life (SD) & $\begin{array}{c}53,3 \\
(16,3)\end{array}$ & $\begin{array}{c}56,7 \\
(26,6)\end{array}$ & $\begin{array}{c}65,0 \\
(21,4)\end{array}$ & $\begin{array}{c}65,6 \\
(20,9)\end{array}$ & $\mathrm{F}=0,010 \mathrm{df}=1$ & $\mathrm{p}=0,919($ group$)$ & $\eta^{2}=0,001$ \\
\hline $\begin{array}{c}\text { Physical } \\
\text { Functioning (SD) }\end{array}$ & $\begin{array}{c}71,3 \\
(13,0)\end{array}$ & $\begin{array}{c}77,3 \\
(15,8)\end{array}$ & $\begin{array}{c}80,4 \\
(21,3)\end{array}$ & $\begin{array}{c}80,9 \\
(18,7)\end{array}$ & $\mathrm{F}=0,276 \mathrm{df}=1$ & $\mathrm{p}=0,610($ group$)$ & $\eta^{2}=0,012$ \\
\hline $\begin{array}{c}\text { Role } \\
\text { Functioning (SD) }\end{array}$ & $\begin{array}{c}61,7 \\
(17,7)\end{array}$ & $\begin{array}{c}60,0 \\
(29,6)\end{array}$ & $\begin{array}{c}67,8 \\
(34,2)\end{array}$ & $\begin{array}{l}73,3 \\
(24,2)\end{array}$ & $\mathrm{F}=1,324 \mathrm{df}=1$ & $\mathrm{p}=0,262($ group $)$ & $\eta^{2}=0,057$ \\
\hline $\begin{array}{c}\text { Emotional } \\
\text { Functioning (SD) }\end{array}$ & $\begin{array}{c}55,8 \\
(26,9)\end{array}$ & $\begin{array}{c}54,2 \\
(26,7)\end{array}$ & $\begin{array}{c}58,9 \\
(31,9)\end{array}$ & $\begin{array}{c}63,9 \\
(32,5)\end{array}$ & $\mathrm{F}=0,864 \mathrm{df}=1$ & $\mathrm{p}=0,363($ group$)$ & $\eta^{2}=0,038$ \\
\hline $\begin{array}{c}\text { Cognitive } \\
\text { Functioning (SD) }\end{array}$ & $\begin{array}{c}\mathbf{7 0 , 0} \\
(\mathbf{3 0 , 2})\end{array}$ & $\begin{array}{l}78,3 \\
(23,6)\end{array}$ & $\begin{array}{c}71,1 \\
(31,2)\end{array}$ & $\begin{array}{c}65,6 \\
(34,2)\end{array}$ & $F=3,322 \mathrm{df}=1$ & $\mathbf{p}=\mathbf{0 , 0 8 2}($ group $)$ & $\square^{2}=\underline{0,131}$ \\
\hline $\begin{array}{c}\text { Social } \\
\text { Functioning (SD) }\end{array}$ & $\begin{array}{c}60,0 \\
(43,2)\end{array}$ & $\begin{array}{c}65,0 \\
(32,8)\end{array}$ & $\begin{array}{c}64,4 \\
(36,1)\end{array}$ & $\begin{array}{c}70,0 \\
(36,3)\end{array}$ & $\mathrm{F}=0,043 \mathrm{df}=1$ & $\mathrm{p}=0,838($ group$)$ & $\eta^{2}=0,002$ \\
\hline Fatigue (SD) & $\begin{array}{c}48,9 \\
(29,3)\end{array}$ & $\begin{array}{c}47,8 \\
(28,2)\end{array}$ & $\begin{array}{c}38,5 \\
(28,8)\end{array}$ & $\begin{array}{c}34,8 \\
(27,8)\end{array}$ & $\mathrm{F}=0,552 \mathrm{df}=1$ & $\mathrm{p}=0,465($ group $)$ & $\eta^{2}=0,024$ \\
\hline $\begin{array}{c}\text { Nausea and } \\
\text { Vomiting (SD) }\end{array}$ & $\begin{array}{l}, 0 \\
(, 0)\end{array}$ & $\begin{array}{c}3,3 \\
(10,5)\end{array}$ & $\begin{array}{c}5,6 \\
(17,4)\end{array}$ & $\begin{array}{c}3,3 \\
(9,3)\end{array}$ & $\mathrm{F}=0,073 \mathrm{df}=1$ & $\mathrm{p}=0,790($ group$)$ & $\eta^{2}=0,003$ \\
\hline Pain (SD) & $\begin{array}{c}38,3 \\
(24,9)\end{array}$ & $\begin{array}{l}41,7 \\
(28,6)\end{array}$ & $\begin{array}{c}36,7 \\
(32,2)\end{array}$ & $\begin{array}{c}40,0 \\
(31,4)\end{array}$ & $\mathrm{F}=0,003 \mathrm{df}=1$ & $\mathrm{p}=0,954$ (group) & $\eta^{2}=0,001$ \\
\hline Dyspnoea (SD) & $\begin{array}{l}23,3 \\
(22,5)\end{array}$ & $\begin{array}{l}23,3 \\
(27,4)\end{array}$ & $\begin{array}{c}33,3 \\
(37,8)\end{array}$ & $\begin{array}{c}26,7 \\
(33,8)\end{array}$ & $F=0,206 \mathrm{df}=1$ & $\mathrm{p}=0,654($ group $)$ & $\eta^{2}=0,009$ \\
\hline Insomnia (SD) & $\begin{array}{c}56,7 \\
(38,7)\end{array}$ & $\begin{array}{c}56,7 \\
(41,7)\end{array}$ & $\begin{array}{c}57,8 \\
(36,7)\end{array}$ & $\begin{array}{c}48,9 \\
(39,6)\end{array}$ & $\mathrm{F}=0,861 \mathrm{df}=1$ & $\mathrm{p}=0,363($ group$)$ & $\eta^{2}=0,038$ \\
\hline Appetite loss (SD) & $\begin{array}{c}20,0 \\
(35,8)\end{array}$ & $\begin{array}{c}23,3 \\
(41,7)\end{array}$ & $\begin{array}{c}11,1 \\
(24,1)\end{array}$ & $\begin{array}{c}4,4 \\
(11,7)\end{array}$ & $F=2,323 d f=1$ & $\mathrm{p}=0,142($ group $)$ & $\eta^{2}=0,096$ \\
\hline Constipation (SD) & $\begin{array}{c}13,3 \\
(32,2)\end{array}$ & $\begin{array}{c}23,3 \\
(31,6)\end{array}$ & $\begin{array}{c}22,2 \\
(27,2)\end{array}$ & $\begin{array}{c}15,6 \\
(21,3)\end{array}$ & $\mathrm{F}=1,733 \mathrm{df}=1$ & $\mathrm{p}=0,202($ group$)$ & $\eta^{2}=0,073$ \\
\hline Diarrhoea (SD) & $\begin{array}{c}3,3 \\
(10,5)\end{array}$ & $10(22,5)$ & $\begin{array}{c}6,7 \\
(18,7)\end{array}$ & $\begin{array}{c}2,2 \\
(8,6)\end{array}$ & $\mathrm{F}=1,939 \mathrm{df}=1$ & $\mathrm{p}=0,178($ group $)$ & $\eta^{2}=0,081$ \\
\hline $\begin{array}{c}\text { Financial } \\
\text { Diffuculties (SD) }\end{array}$ & $\begin{array}{c}30,0 \\
(42,9)\end{array}$ & $\begin{array}{c}30,0 \\
(36,7)\end{array}$ & $\begin{array}{c}26,7 \\
(38,2)\end{array}$ & $\begin{array}{c}28,6 \\
(38,9)\end{array}$ & $\mathrm{F}=0,702 \mathrm{df}=1$ & $\mathrm{p}=0,412($ group $)$ & $\eta^{2}=0,032$ \\
\hline Body Image (SD) & $\begin{array}{c}52,5 \\
(35,1)\end{array}$ & $\begin{array}{c}53,9 \\
(32,3)\end{array}$ & $\begin{array}{c}62,8 \\
(36,3)\end{array}$ & $\begin{array}{c}66,7 \\
(36,2)\end{array}$ & $\mathrm{F}=0,301 \mathrm{df}=1$ & $\mathrm{p}=0,589($ group $)$ & $\eta^{2}=0,013$ \\
\hline $\begin{array}{c}\text { Sexual } \\
\text { Functioning (SD) }\end{array}$ & $\begin{array}{c}77,1 \\
(30,8)\end{array}$ & $\begin{array}{c}77,1 \\
(19,8)\end{array}$ & $\begin{array}{c}72,2 \\
(33,7)\end{array}$ & $\begin{array}{c}81,0 \\
(24,3)\end{array}$ & $\mathrm{F}=1,341 \mathrm{df}=1$ & $\mathrm{p}=0,262($ group $)$ & $\eta^{2}=0,069$ \\
\hline $\begin{array}{c}\text { Sexual } \\
\text { Enjoyment (SD) }\end{array}$ & $\begin{array}{c}66,7 \\
(47,1)\end{array}$ & $\begin{array}{c}44,4 \\
(19,2)\end{array}$ & $\begin{array}{c}50,0 \\
(43,0)\end{array}$ & $\begin{array}{c}50,0 \\
(43,0)\end{array}$ & $\mathrm{F}=2,400 \mathrm{df}=1$ & $\mathrm{p}=0,261($ group$)$ & $\left(\eta^{2}=0,545\right)$ \\
\hline $\begin{array}{c}\text { Future } \\
\text { Perspective (SD) }\end{array}$ & $\begin{array}{c}50,0 \\
(28,3)\end{array}$ & $\begin{array}{c}56,7 \\
(31,6)\end{array}$ & $\begin{array}{c}46,7 \\
(41,4)\end{array}$ & $\begin{array}{c}48,9 \\
(39,6)\end{array}$ & $\mathrm{F}=0,373 \mathrm{df}=1$ & $\mathrm{p}=0,548($ group $)$ & $\eta^{2}=0,017$ \\
\hline $\begin{array}{l}\text { Systemic Therapy } \\
\text { Side Effects (SD) }\end{array}$ & $\begin{array}{c}34,8 \\
(19,8)\end{array}$ & $\begin{array}{c}33,3 \\
(19,0)\end{array}$ & $\begin{array}{c}27,9 \\
(14,9)\end{array}$ & $\begin{array}{c}27,0 \\
(19,3)\end{array}$ & $\mathrm{F}=0,015 \mathrm{df}=1$ & $\mathrm{p}=0,904($ group$)$ & $\eta^{2}=0,001$ \\
\hline $\begin{array}{l}\text { Breast Symptoms } \\
\text { (SD) }\end{array}$ & $\begin{array}{c}15,8 \\
(19,0)\end{array}$ & $\begin{array}{l}21,7 \\
(22,3)\end{array}$ & $\begin{array}{c}25,0 \\
(21,1)\end{array}$ & $\begin{array}{c}28,9 \\
(24,6)\end{array}$ & $\mathrm{F}=0,005 \mathrm{df}=1$ & $\mathrm{p}=0,942($ group $)$ & $\eta^{2}=0,001$ \\
\hline $\begin{array}{l}\text { Arm Symptoms } \\
\text { (SD) }\end{array}$ & $\begin{array}{c}33,3 \\
(29,6)\end{array}$ & $\begin{array}{c}30,0 \\
(29,7)\end{array}$ & $\begin{array}{c}37,0 \\
(27,1)\end{array}$ & $\begin{array}{c}41,5 \\
(25,7)\end{array}$ & $\mathrm{F}=1,494 \mathrm{df}=1$ & $\mathrm{p}=0,234($ group$)$ & $\eta^{2}=0,064$ \\
\hline $\begin{array}{l}\text { Upset by Hair loss } \\
\text { (SD) }\end{array}$ & $\begin{array}{l}66,7 \\
(, 0)\end{array}$ & $\begin{array}{l}33,3 \\
(, 0)\end{array}$ & $\begin{array}{c}61,1 \\
(39,0)\end{array}$ & $\begin{array}{c}53,3 \\
(47,7)\end{array}$ & $\mathrm{F}=1,143 \mathrm{df}=1$ & =0,397 (group) & $\left(\eta^{2}=0,364\right)$ \\
\hline
\end{tabular}

* effect size related to the effect of the factor "group"

most participants of the MBSR group did not answer the EORTCquestionnaire as to questions concerning sexual enjoyment and only one participant could report to have still pleasant sexual experiences.

This result of a disturbed acceptance of parts of the body could be an effect of the attention and self-reflection which is induced by MBSR training. Women might have located unconsciously their fear of breast cancer in their body experience. Therefore, we propose individual psychotherapy to develop confidence in their own body sensations after cancer treatment.

Further studies are necessary because of the small sample in this study.

\section{References}

1. Mattes J (2017) Awareness Culture and Health: A Prospective, Randomized and Controlled Study of Spiritual Practice in Breast Cancer Patients. Marburg, Tectum Verlag, ISBN 978-3-8288-3907-6.

Copyright: (C2019 Mattes JF. This is an open-access article distributed under the terms of the Creative Commons Attribution License, which permits unrestricted use, distribution, and reproduction in any medium, provided the original author and source are credited. 